\title{
A resting state network in the motor control circuit of the basal
} ganglia

\author{
Simon Robinson*1,4, Gianpaolo Basso ${ }^{1}$, Nicola Soldati2 ${ }^{2}$ Uta Sailer ${ }^{3}$, \\ Jorge Jovicich $^{1}$, Lorenzo Bruzzone ${ }^{2}$, Ilse Kryspin-Exner ${ }^{3}$, Herbert Bauer ${ }^{3}$ and \\ Ewald Moser 4,5
}

\author{
Address: ${ }^{1}$ Functional Neuroimaging Laboratory, Center for Mind/Brain Sciences, University of Trento, Trento, Italy, ${ }^{2}$ Telecommunication \\ Engineering, University of Trento, Trento, Italy, ${ }^{3}$ Faculty of Psychology, University of Vienna, Liebiggasse 5, 1010 Vienna, Austria, ${ }^{4} \mathrm{MR}$ Center of \\ Excellence, Medical University of Vienna, Lazarettgasse 14, 1090 Vienna, Austria and ${ }^{5}$ Center for Biomedical Engineering and Physics, Medical \\ University of Vienna, Währinger Gürtel 18-20, 1090 Vienna, Austria \\ Email: Simon Robinson* - simon.robinson@meduniwien.ac.at; Gianpaolo Basso - gianpaolo.basso@unitn.it; \\ Nicola Soldati - nicola.soldati@unitn.it; Uta Sailer - uta.sailer@univie.ac.at; Jorge Jovicich - jorge.jovicich@unitn.it; \\ Lorenzo Bruzzone - lorenzo.bruzzone@ing.unitn.it; Ilse Kryspin-Exner - ilse.kryspin-exner@univie.ac.at; \\ Herbert Bauer - herbert.bauer@univie.ac.at; Ewald Moser - ewald.moser@meduniwien.ac.at \\ * Corresponding author
}

Published: 23 November 2009

BMC Neuroscience 2009, 10:137

This article is available from: http://www.biomedcentral.com//47/-2202/10/I37

(c) 2009 Robinson et al; licensee BioMed Central Ltd.

This is an Open Access article distributed under the terms of the Creative Commons Attribution License (http://creativecommons.org/licenses/by/2.0), which permits unrestricted use, distribution, and reproduction in any medium, provided the original work is properly cited.
Received: 13 February 2009

Accepted: 23 November 2009

\begin{abstract}
Background: In the absence of overt stimuli, the brain shows correlated fluctuations in functionally related brain regions. Approximately ten largely independent resting state networks (RSNs) showing this behaviour have been documented to date. Recent studies have reported the existence of an RSN in the basal ganglia - albeit inconsistently and without the means to interpret its function. Using two large study groups with different resting state conditions and MR protocols, the reproducibility of the network across subjects, behavioural conditions and acquisition parameters is assessed. Independent Component Analysis (ICA), combined with novel analyses of temporal features, is applied to establish the basis of signal fluctuations in the network and its relation to other RSNs. Reference to prior probabilistic diffusion tractography work is used to identify the basal ganglia circuit to which these fluctuations correspond.
\end{abstract}

Results: An RSN is identified in the basal ganglia and thalamus, comprising the pallidum, putamen, subthalamic nucleus and substantia nigra, with a projection also to the supplementary motor area. Participating nuclei and thalamo-cortical connection probabilities allow this network to be identified as the motor control circuit of the basal ganglia. The network was reproducibly identified across subjects, behavioural conditions (fixation, eyes closed), field strength and echo-planar imaging parameters. It shows a frequency peak at $0.025 \pm 0.007 \mathrm{~Hz}$ and is most similar in spectral composition to the Default Mode (DM), a network of regions that is more active at rest than during task processing. Frequency features allow the network to be classified as an RSN rather than a physiological artefact. Fluctuations in this RSN are correlated with those in the task-positive frontoparietal network and anticorrelated with those in the DM, whose hemodynamic response it anticipates. 
Conclusion: Although the basal ganglia RSN has not been reported in most ICA-based studies using a similar methodology, we demonstrate that it is reproducible across subjects, common resting state conditions and imaging parameters, and show that it corresponds with the motor control circuit. This characterisation of the basal ganglia network opens a potential means to investigate the motor-related neuropathologies in which the basal ganglia are involved.

\section{Background}

A number of studies dating back to 1995 have shown that when subjects are not engaged in processing externally directed tasks or time-varying stimuli - when they are, from a behavioural perspective, at rest - MR images of the brain show correlated, low frequency fluctuations in functionally related areas. This has been interpreted as indicating functional connectivity between regions [1-5]. A number of distinct, largely independent assemblies, or Resting State Networks (RSNs) have been discovered since using semi-exploratory and exploratory analysis methods.

It has recently been shown that RSN fluctuations explain not only inter-trial variation in the BOLD response [6] but also behaviour [7] and that some RSNs are disturbed in pathologies such as Alzheimer's disease (e.g. [8]). This has fuelled efforts to better characterise these networks through behavioural manipulation $[9,10]$ and by their interdependence on other networks $[11,12]$, not only to improve experiment design but also to better understand healthy brain function and a range of neurological and psychiatric conditions.

The first RSNs were discovered using functional connectivity analysis, in which correlation is performed between the time course in a seed voxel or region and that in other voxels, in order to reveal areas whose activity is coupled. The discovery that functional connectivity could be observed between ipsilateral and contralateral sensorimotor regions [1] was rapidly followed by similar observations for visual and auditory areas [13], the amygdala [4] and the thalamus and hippocampus [14]. It was later discovered that the group of regions which have come to be known as the Default Mode network, which had been observed to be more active during rest periods than during task processing [15-17], also show fluctuations characteristic of RSNs during rest periods [5]. The development of group Independent Component Analysis methods allowed a fully exploratory approach to identifying RSNs [18-20], and led to the elucidation of other networks in posterior parietal areas, lateralised left and right frontoparietal regions, the anterior temporal lobe, cerebellum and limbic lobe $[9,21-23]$. To date, approximately 10 RSNs have been reproducibly identified $[9,23]$.

There is no a priori model in functional connectivity analysis, but a seed voxel (or ROI) time-course is selected by the experimenter. This process leaves the approach prone to omission unless correlations are computed between a large number of regions (see, e.g., Achard et al. [24]), and also to weakening by inter-subject variation if seed regions are defined on the basis of template anatomy rather than individual function. Activation results from functional tasks may be used to define seed regions, but this becomes impractical if many networks are to be analysed in the same data. The sensitivity of the analysis is reduced if subregions of the same network are separated according to a hypothesis about possible division of function. Alternatively, erroneous conclusions may be drawn about regions functionally connected if seed regions are used that subsume areas which contribute to different networks. In addition, the signal in a seed region comprises many sources, of both neuronal and non-neuronal origin (such as scanner drift). While the inclusion of motion parameters in the general linear model as well as regressors for global, ventricular and white matter signals allows these effects to be reduced $[11,25]$, it has proved difficult to separate a number of physiological artefacts from RSNrelated fluctuations, such as those arising from changes in respiratory rate from the Default Mode [26]. Likewise, the subtraction of global signal to try to mitigate this problem may introduce artificial anticorrelation relationships between component time courses $[25,27,28]$, making study of the interrelation between networks problematic. As a genuinely exploratory method, ICA yields potentially interesting components in the context of the other signal fluctuations in the data and is generally capable of separating overlapping signal sources of physiological and neuronal origin [29]. As such is it well suited to an exploratory analysis aimed of RSNs and their interrelations.

Most RSN findings to date relate to the cerebral cortex. There are reports, mostly restricted to the functional connectivity literature, of correlated fluctuations between isolated subcortical structures, including the amygdala [4] and the thalamus and hippocampus [14]. The involvement of the hippocampus in a (usually subdivided) sensory-auditory RSN [22] and in the Default Mode has been noted [22] as has the inclusion of number of thalamic nuclei in the medial visual, auditory and medial frontal RSNs [21]. Jafri et al. also identify elements of the basal ganglia, albeit in a predominantly cortical frontal parietal subcortical network [12]. We recently reported the existence of an RSN in the basal ganglia and thalamus with 
weak projections to supplementary motor areas [30]. This network overlaps substantially with that identified in a contemporaneous functional connectivity study by $\mathrm{Di}$ Martino et al. (corresponding to seeds in the dorsal and caudal putamen) [31], and a subsequent incidental noting of the same network by Damoiseaux et al. [32]. The network was not present in any other ICA-based study of which we are aware (e.g. [9,21-23]).

These scant and inconsistent reports leave the consistency and role of this network open to question. A recent dispute indicates that apparently subtle variations in behavioural condition can give rise to the appearance of spontaneous activation [33]. Both the Di Martino et al. study [31] and our initial report [30] were based on subjects visually fixating, unlike the majority of RSN studies, in which subjects had their eyes closed, and in which the basal ganglia RSN was not observed. In that light, it seems pertinent to investigate the conditions under which this network is manifest. The origin of these signal fluctuations likewise needs to be established; whether they are neuronal resting-state fluctuations or a physiological artefact. If the origins of these network are neuronal, to which of the many parallel basal ganglia circuits and functions do they correspond, and what relationship do they have to other resting state networks?

The basal ganglia consist of four nuclei; the striatum (which is subdivided into the caudate nucleus and putamen), the globus pallidus or pallidum, the substantia nigra and the subthalamic nucleus [34]. Originally viewed as motor structures, tracing studies in the monkey suggested that the striatum could be divided into two networks, sensorimotor and associative [35]. A more recent model has suggested that the diverse functions in which the basal ganglia are involved are expressed through five parallel segregated circuits; motor, oculomotor, dorsolateral prefrontal, lateral orbitofrontal and anterior cingulate [36]. Each of these receives input from a number of functionally related neocortical regions (e.g. in the case of the motor circuit, the supplementary motor area, arcuate premotor area, motor cortex and somatosensory cortex) and outputs to a single frontal region (e.g. in the motor circuit, the supplementary motor area). In the current view, the basal ganglia are envisaged as comprising just three distinct functional assemblies; the sensorimotor, the associative and the limbic [37]. The fact that each of the corresponding circuits involves distinct regions of striatum, pallidum, substantia nigra, thalamus and cortex offers the possibility - within the limits of activation localisation - of identifying the circuit to which the network corresponds, and with that, its function.

We examine resting-state fluctuations in the thalamus and basal ganglia using two common resting state conditions, two large, independent study groups and a fast EPI protocol optimised for structures with a short T2* $[38,39]$, combined with high field strength. We apply group ICA tools to identify this network in studies using fixation and eyes closed conditions, and refer to known basal ganglia circuits and probabilistic tractography to identify the function subsumed by this network. Temporal features of independent components are used in a novel classifier to distinguish RSNs from physiological artefacts, and functional network connectivity is applied to elucidate the relationship between the basal ganglia resting state network and the Default Mode.

\section{Results}

We find a resting state network involving the thalamus and a large portion of the basal ganglia in groups studied under both the eyes open and fixation conditions, and a temporally coherent network focussed on the caudate in the fixation study only.

The distributed basal ganglia network is symmetric and incorporates the pallidum, putamen, subthalamic nucleus and substantia nigra, as well as the thalamus, with weaker extensions to the transverse temporal gyrus and the supplementary motor area (SMA). The network is illustrated as it appears in the MELODIC analysis of the fixation study group of 26 subjects in Figure 1. Previous studies employing PICA have employed a posterior probability threshold of $\mathrm{P}>0.5$ using the mixture model approach $[18,21-23,32,40]$. Non-outlined slices in Figure 1 derive from ICA of downsampled, smoothed data and are thresholded using a more stringent threshold of $\mathrm{P}>0.99$. To enable more exact localisation of activation foci in the region of the substantia nigra and subthalamic nucleus, results based on unsmoothed, non-resampled data have been illustrated for that region (the top row of Figure 1, slices outlined in brown), and are thresholded at the canonical level of $\mathrm{P}>0.5$. The same data and threshold were used for the slice showing activation in the SMA (Figure 1C). The component is overlaid on the mean anatomical (MPRAGE) image for the group. To aid orientation the location of selected slices (labelled A-C) is indicated on the MNI template $\mathrm{T}_{2}$ image (right panel). The entirety of the network is shown in Additional File 1 (downsampled data, thresholded at $\mathrm{P}>0.5$ ).

The basal ganglia network is also present in the analyses of the two subgroups of 13 subjects in the fixation study (Rows 2 and 3 respectively in Figure 2) and in the eyes closed study in which a separate group of subjects were scanned with their eyes closed (Row 4 in the same figure). In all cases, it appears substantially unvaried from its manifestation in the whole group analysis (Row 1). The eyes closed study included both male and female subjects and was conducted at $4 \mathrm{~T}$ with a very different EPI proto- 


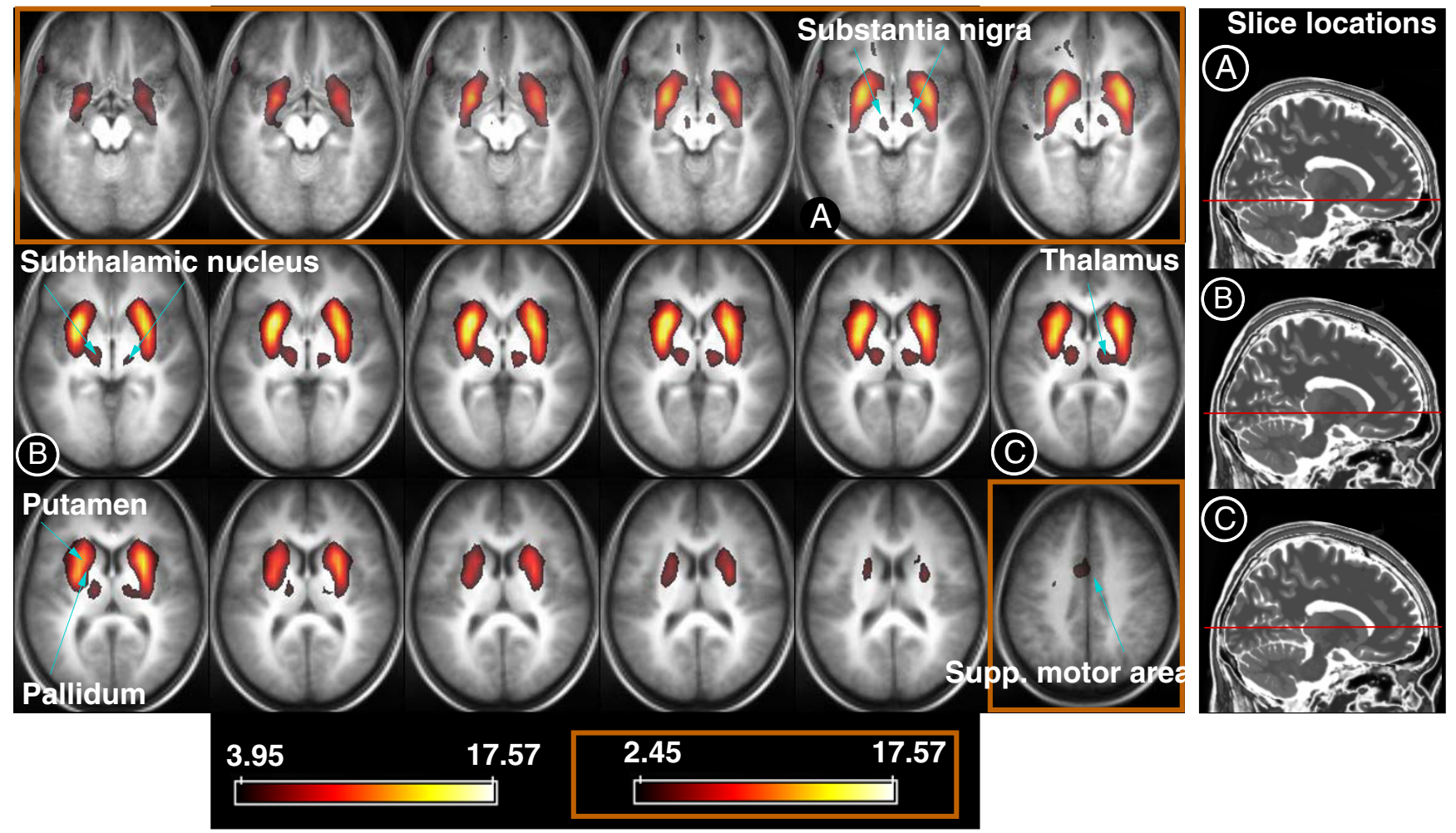

Figure I

The basal ganglia resting state network. It comprises, bilaterally, the pallidum, putamen, substantia nigra, subthalamic nucleus and thalamus. In the overlay, which is on the mean anatomical image for the group, maps of $z$ statistics are thresholded at $\mathrm{P}>0.99$ (see colorbar on bottom left) other than slices outlined in brown, which are thresholded at $\mathrm{P}>0.5$ (see colorbar on bottom right in brown box). All slices are shown thresholded at $\mathrm{P}>0.5$ in Additional File $\mathrm{I}$.

col, showing the network to be reproducible across a wide range of factors; resting state condition (fixation and eyes closed), gender and measurement parameters, including field strength and repetition time.

In addition to the basal ganglia network, in which one element of the striatum - the caudate nucleus - is not present, a separate component was identified in the fixation study data, which consists of the caudate and, much more weakly, dorsolateral prefrontal cortex and posterior parietal cortex (Figure 3 ). This is absent in the study in which subjects had their eyes closed.

A public-access atlas of thalamo-cortical connection probabilities http://www.fmrib.ox.ac.uk/connect/ based on results of a prior study by Behrens et al. [41] shows that, for the basal ganglia the thalamic activation foci identified connect with high probability to motor areas. For the focus with MNI co-ordinates $(13,-20,0)$ these probabilities take the values of 0.68 to the primary motor, 0.44 to the sensory and 0.28 to the pre-motor cortex. For the focus at $(-13,-20,0)$ the respective probabilities are $0.44,0.38$ and 0.43 . The probabilities of the primary thalamic con- nections being to none-motor regions (occipital, prefrontal, posterior parietal and temporal cortices) are below 0.2 for both foci.

All the other resting state networks reported in the ICAbased resting state literature to date $[9,21-23,32]$ were also identified in the MELODIC analysis of the fixation study (Table 1). Despite its reproducibility, the basal ganglia network emerges as one of the weakest in these data in terms of the percentage of variance in the data explained by each component $(0.13 \%)$. The only subcortical elements present in other networks were the hippocampus in the Default Mode (consistent with e.g. [15]) and the weak presence of the substantia nigra in the posterior parietal network.

The same resting state networks, including the basal ganglia resting state network, were identified in the GIFT results, which were used to extract single-subject temporal responses for the assessments of temporal and frequency features that follow. The spatial map of the basal ganglia component identified in the GIFT analysis is shown in Additional File 2. 


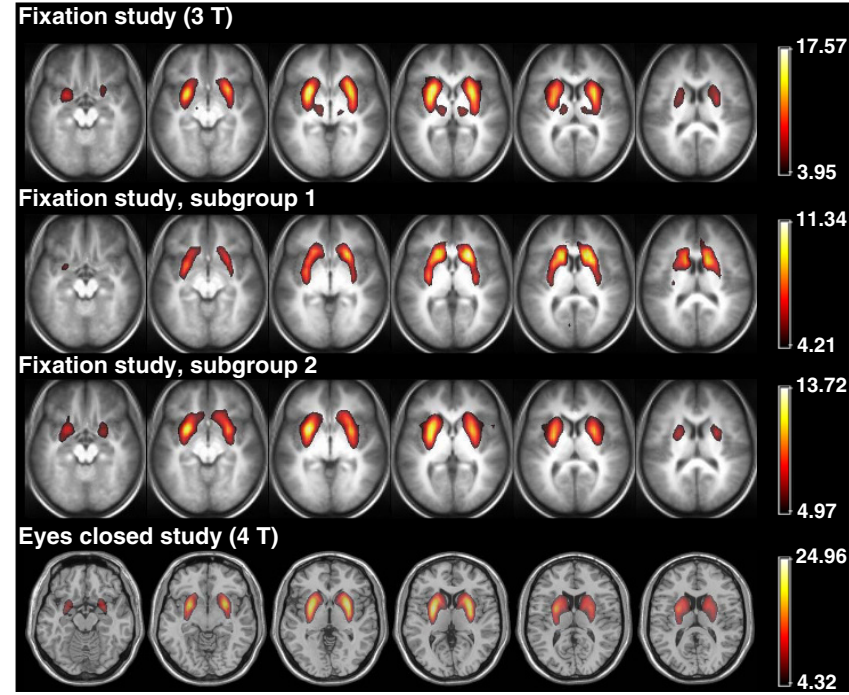

Figure 2

The reproducibility of the basal ganglia resting network. The top row shows selected slices from the main study with all subjects. The second and third rows illustrate results for subgroups I and 2 respectively. The bottom row shows the same network as it appears in the eyes closed study (eyes closed, $4 \mathrm{~T}$, both male and female participants). All RSN z maps are thresholded at $\mathrm{P}>0.99$.

The mean frequency spectrum of the basal ganglia network is illustrated in Figure 4, in which the grey band indicates one standard deviation from the mean across subjects. On average, the position of the maximum in the spectral distribution was at $0.025 \pm 0.007 \mathrm{~Hz}$.

To investigate the possibility of causal relationships existing between the basal ganglia and other RSNs (similar to those which have been demonstrated between the Default Mode and the lateral parietal networks $[5,11,42,43])$, the mean correlation was calculated between component time courses for the basal ganglia RSN and other RSNs over all subjects. The most significant results are listed in Table 2. The basal ganglia RSN was anti-correlated with the Default Mode $\left(-0.27 \pm 0.24, \mathrm{p}=8.4 \times 10^{-6}\right)$ and positively correlated, to a similar degree, with both the right and left lateral fronto-parietal networks $(0.20 \pm 0.26, \mathrm{p}=$ $5.8 \times 10^{-4}$ and $0.24 \pm 0.18, \mathrm{p}=9.4 \times 10^{-7}$, respectively). These values may be compared with those obtained in this analysis between the Default Mode and the lateral frontoparietal networks; $-0.19 \pm 0.34, \mathrm{p}=0.011$ (right) and -0.19 $\pm 0.21, \mathrm{p}=1.6 \times 10^{-4}$ (left). That is, the correlations between the basal ganglia RSN and the Default Mode, and the basal ganglia RSN and lateral parietal networks are consistent with those between the Default Mode and the lateral parietal networks but are stronger and more significant. Functional network connectivity analysis [12] applied to the basal ganglia RSN and the Default mode -

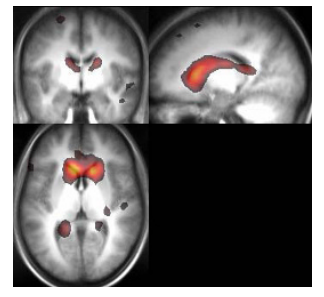

Figure 3

A component showing caudate activity in the main study. The overlay of $z$ statistics is thresholded at $P>0.5$.

performed for 20 randomly-composed groups of 13 subjects from the population of 26 subjects - showed significant correlation between the two networks for all 20 groups. In 18 of these the hemodynamic response in the basal ganglia was in anticipation of that in the Default Mode (by an average of $0.56 \pm 0.51 \mathrm{~s}$ ), in 2 it lagged behind it.

A number of components were identified by experienced raters (SR and GB) as being of physiological origin based on their spatial distribution. These included components of vascular origin located in the Circle of Willis, distributed grey matter components arising from respiration rate variation of the type reported by Birn et al. [29], and CSF flow between the ventricles. The mean frequency spectrum of the basal ganglia RSN is also plotted in Figure 5 along with the frequency spectra of components of established RSN origin and physiological components. Spectral power was focussed at lower frequencies for RSNs than physiological components. The two frequency features described in the Methods section, "Dynamic Range" and "Low to High Power Ratio", are plotted for RSNs and physiological component spectra in Figure 6. The known RSNs are well separated from physiological components. The basal ganglia component clusters with known RSNs.

The "Dynamic Range" feature afforded 93\% accuracy in distinguishing resting state networks and physiological components, with 0 false negatives (no RSNs incorrectly identified as a physiological signal source) and 1 false positive (1 physiological component labelled incorrectly as an RSN). The basal ganglia component was classified as an RSN.

\section{Discussion}

We detail the structures contributing to a recently reported resting state network in the thalamus and basal ganglia. By using a high field, high BOLD sensitivity experiment design and high resolution analysis, we show that it encompasses not only the thalamus, pallidum, putamen and transverse temporal gyrus - as has been previously 
Table I: The RSNs identified in the MELODIC analysis of the main study group, the names adopted in this work, the relative strength of the components and correspondence to RSNs identified in other studies (the bracketed suffixes $L$ and $R$ indicate that the component identified here corresponds to either the left or right hand side of the network referred to).

\begin{tabular}{|c|c|c|c|}
\hline Component Number & RSN Name & Percentage of explained variance & $\begin{array}{l}\text { Labeling in [(Calhoun et al., 2008), } \\
\text { (Damoiseaux et al., 2006), (Beckmann et } \\
\text { al., 2005), (De Luca et al., 2006)] } \\
\text { respectively. }\end{array}$ \\
\hline 10 & Medial visual & 3.81 & {$[D, E,(a)]$,} \\
\hline 11 & Motor & 3.59 & {$[B, F,(d), R S N 3]$} \\
\hline 12 & Cerebellum & 3.31 & {$[\mathrm{H},,]$,} \\
\hline 15 & Lateral visual & 2.67 & {$[\mathrm{~F}, \mathrm{~A},(\mathrm{~b}), \mathrm{RSN} \mathrm{I}]$} \\
\hline 20 & Posterior parietal & 2.18 & {$[\mathrm{C}, \mathrm{H}]$,} \\
\hline 23 & Left lateral fronto-parietal & 1.93 & {$[\mathrm{E}, \mathrm{C},(\mathrm{h}), \mathrm{RSN} 4(\mathrm{~L})]$} \\
\hline 28 & Temporal lobe & 1.15 & {$[\mathrm{I}, \mathrm{I},(\mathrm{c}), \mathrm{RSN} 3]$} \\
\hline 31 & Medial frontal & 0.85 & {$[\mathrm{~J},(\mathrm{f}), \mathrm{RSN} 2]$} \\
\hline 36 & Default Mode & 0.53 & {$[\mathrm{~A}, \mathrm{~B},(\mathrm{e}), \mathrm{RSN} 2]$} \\
\hline 40 & Limbic lobe & 0.28 & {$[\mathrm{~L},,]$,} \\
\hline 41 & Basal ganglia & 0.13 & \\
\hline 43 & Right lateral fronto-parietal & 0.08 & {$[\mathrm{~K}, \mathrm{D},(\mathrm{g}), \mathrm{RSN} 4(\mathrm{R})]$} \\
\hline 52 & Anterior temporal lobe & $\dagger$ & {$[\mathrm{G},,]$,} \\
\hline
\end{tabular}

$\dagger$ - no valid value was obtained for this component.

noted - but also the substantia nigra and subthalamic nucleus, allowing the basal ganglia circuit to which it corresponds to be identified. Despite its non-observation in most resting-state studies to date $[9,21-23]$ it was found to be reproducible across subjects and MR measurement parameters and is evident in both the eyes closed and fixation resting state conditions. The network is positively correlated with the left and right lateral fronto-parietal (also called attention or task-positive) networks, and anticorrelated with the Default Mode, whose hemodynamic response it anticipates. The peak frequency and spectral characteristics are similar to those of other RSNs, distinct from physiological fluctuations, and allow it to be classi-

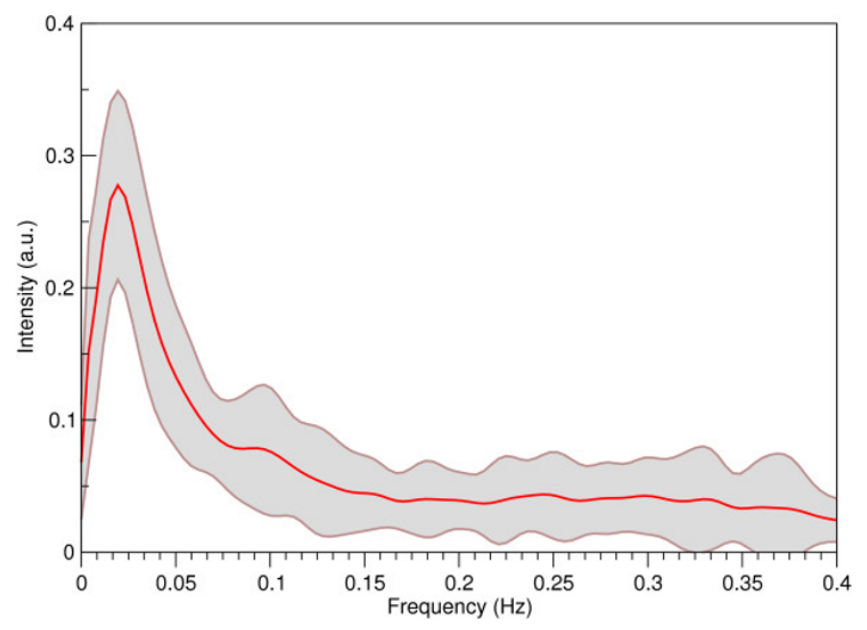

Figure 4

Mean frequency spectrum of the network (over subjects). The boundaries of the shaded area denote one standard deviation on the mean. fied as an RSN using the unsupervised classifier described. We proceed to identify which of the parallel segregated basal ganglia circuits this network corresponds to based on participating regions.

Because of their historical significance as motor structures, the best studied basal ganglia network is the motor circuit. Cortical input from precentral motor areas, postcentral somatosensory areas, the arcuate premotor area and the supplementary motor areas projects dominantly to the putamen. The putamen sends projections to the interior segment of the pallidum and on to particular thalamic nuclei (the direct pathway) as well as the internal segment of the globus pallidus via the caudolateral substantia nigra to the thalamus (the indirect pathway). Outputs from the thalamus project to the supplementary motor area, the motor cortex and arcuate premotor area, probably in distinct subcircuits [34]. The observed resting state network corresponds well with the motor circuit; the caudate is notably absent, and the putamen constitutes the focus of activation. The activated regions identified as substantia nigra are consistent with coordinates derived from stereotactic electrophysiological studies [44]. The presence of the substantia nigra suggests that the resting state network corresponds either solely to the indirect pathway of the motor circuit, which acts to inhibit movement, or both the indirect and direct pathways. Weak activation in the supplementary motor area is likewise consistent with the motor circuit. Myeloarchitectonics suggest that the thalamic activation corresponds to the ventro-lateral thalamic nucleus, which a prior DTI study has shown to connect dominantly to motor regions [41]. In fact, endogenous BOLD fluctuations in this part of the thalamus have been found to be strongly correlated with motor and pre- 
Table 2: Mean correlation between the basal ganglia component time course and the time courses of other networks, across subjects, listing the most significant mean correlations.

\begin{tabular}{lrr}
\hline Network Names & Correlation & Standard deviation \\
\hline Basal ganglia, Default Mode & -0.27 & 0.24 \\
Basal ganglia, right lateral fronto-parietal & 0.20 & $8.4 \times 10^{-6}$ \\
Basal ganglia, left lateral fronto-parietal & 0.24 & $5.8 \times 10^{-4}$ \\
& & $9.4 \times 10^{-7}$ \\
*Default Mode, right lateral fronto-parietal & -0.19 & 0.18 \\
*Default Mode, left lateral fronto-parietal & -0.19 & 0.29 \\
\end{tabular}

*These networks possess an anti-correlation well-documented in the literature, and are presented for comparison.

motor areas by Zhang et al. ([45] - supplementary material) as well as the whole putamen.

As well as there being good agreement between the areas observed in this RSN and the motor circuit, there is disparity between the principle sites of input to the striatum and the other circuits. The caudate provides input to the oculomotor, dorsolateral prefrontal and lateral orbitofrontal circuits and the ventral striatum provides input to the anterior cingulate circuit. The cortical regions to which the circuits send output are the frontal eye fields for the oculomotor circuit and the dorsolateral prefrontal cortex, the lateral orbitofrontal cortex and the anterior cingulate area for those circuits respectively.

No components were identified corresponding to the associative and limbic thalamo-cortical loops [36]. The question arises of whether it is to be expected that an RSN exists for each of the tripartite or Alexander subdivisions of the basal ganglia, and whether basal ganglia RSNs (if present) would be expected to correspond exactly with cir-

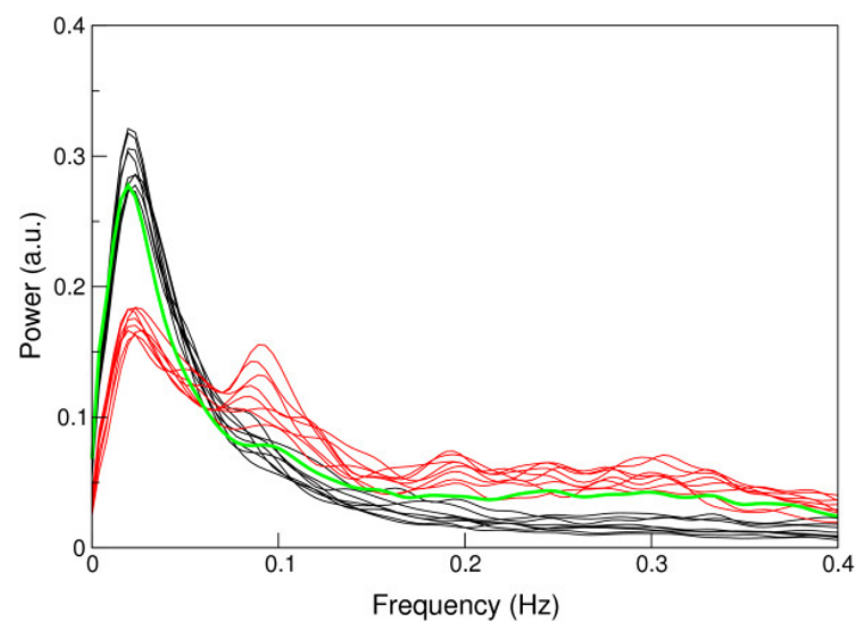

Figure 5

Comparison of the mean spectral distribution of the basal ganglia RSN component over subjects (green) with the spectral distributions of documented RSNs (black) and physiological components (red). cuits that have been established from anatomical and afferent projections from the cortex. Looking outside the basal ganglia, we know that whilst RSNs reflect functional task networks, it is not the case that there is an RSN for every functional network defined by task or anatomy. Where there are spontaneous fluctuations in networks at rest, other studies have shown that there is not complete correspondence with the network as defined by anatomy or tasks. The major anatomical afferents from the cortex suggest a functional partitioning of the putamen, with a minor portion classified as associative and limbic, and the majority as being sensorimotor. Zhang et al. found functional connectivity between the motor and premotor cortex and almost the entire putamen [45], however, as observed here. The temporal cortex also displayed a much weaker correlation with the caudal-ventral putaminal region, a finding not consistent with the classical striate nucleus tripartite subdivision. In addition, when looking at RSNs involving associative cortical area, such as the

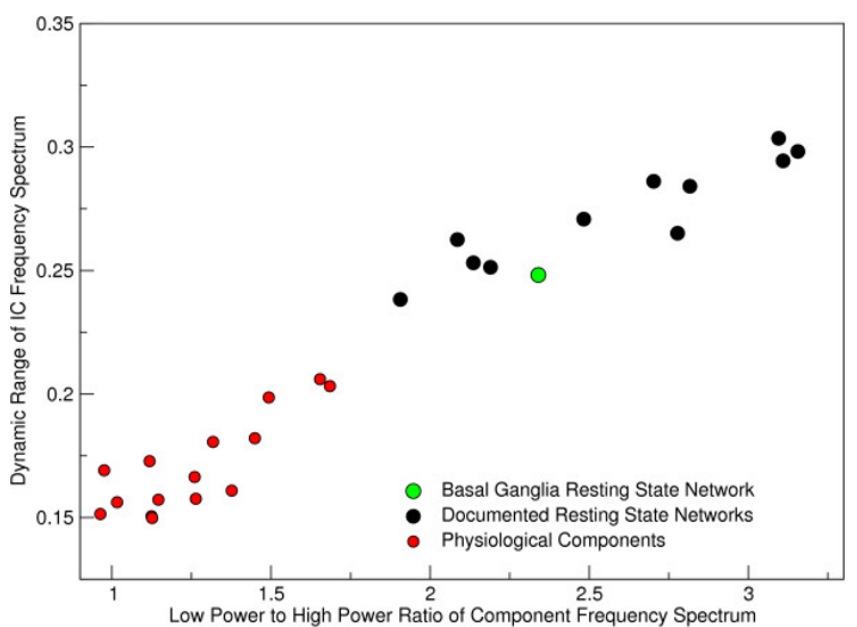

Figure 6

Plot of the space of the spectral features described in the text, indicating separation of the RSNs documented to date (black) from components reflecting physiological signal fluctuations (red). The frequency properties of the basal ganglia (green) show it to group with other RSNs. 
Default Mode, ventral attention, dorsal attention and executive control networks, only a portion of the caudate nucleus participates, instead of the entire associative subdivision of the striate nucleus.

Our hybrid simulations suggest that ICA is capable of separating circuits which overlap to some extent, but which also have non-overlapping elements and different temporal behaviour (Additional File 3). Further evidence of this is provided by the fact that some brain regions (such as the thalamus) are present in multiple networks. The most likely explanations for not finding RSNs corresponding to the associative and limbic thalamo-cortical circuits is, therefore, that they either do not show spontaneous lowfrequency BOLD fluctuations or that these fluctuations are below the sensitivity of this study.

Di Martino et al. have reported a resting state functional connectivity analysis focussing on the striatum [31] independent of our preliminary reporting of these results [30]. A gender-mixed group of subjects were studied while fixating on the word "Relax" in a study with a sensitivity likely to be equivalent to that here; applying a similar, relatively short TE EPI protocol and comparable number of total image volumes (6895 c.f. 7800 here). The connectivity results obtained for seeds in the dorsal and caudal putamen in the Di Martino et al. study is similar to the RSN observed by us [30], although the involvement of the substantia nigra (which contributes substantially to the motor circuit attribution) was not reported. Also, the dorsal putamen seeds indicated correlated activity in the anterior cortex cingulate, which relate to executive function, indicating some mixing of fluctuations relating to motor and executive control circuits. The basal ganglia RSN reported here was recently noted as an incidental finding by Damoiseaux et al. [32], adding independent verification of these results.

The conditions under which this resting state data were acquired were similar to those used in a number of previous studies which also applied group ICA approaches [9,21-23]. The question then arises as to why this network was not reported in those studies, but has been observed only here and in the most recent publication by Damoiseaux et al. [32]. The basal ganglia resting state network was one of the weakest identified in this study, measured in terms of the percentage of total variance in the data it explains. The sensitivity of this study is likely to be higher than that in the studies cited due to the high field strength (3 T), relatively large number of subjects (26) and short repetition time ( $1 \mathrm{~s})$. The echo time of $28 \mathrm{~ms}$ is quite short and well matched to the $\mathrm{T}_{2}{ }^{*}$ of basal ganglia structures at $3 \mathrm{~T}$ [38], yielding optimal BOLD sensitivity. $\mathrm{T}_{2}{ }^{*}$ is shorter in the basal ganglia than is typical in the cortex due high iron concentration [46]. While it is likely that, other than reference [32], previous studies were not sufficiently sensitive to detect this network, it is also possible that it was simply overlooked in the wealth of components.

Previous studies using functional connectivity analyses have demonstrated an anticorrelation relationship between the Default Mode and the dorsal attention network in the resting state $[5,11,42,43]$, which has been interpreted as indicating an interplay between modes thought to reflect stimulus-independent thought and goal-driven activity. These anticorrelation findings have been recently called into question, however, as global signal subtraction performed as a pre-processing step to reduce the influence of physiological noise and scanner drift itself introduces anticorrelations [27,28]. The approach taken here is not subject to these problems. We calculated the correlation between independent component time courses, with no prior global signal subtraction. The most significant results were as follow. The anticorrelation finding reported previously between the Default Mode and the dorsal attention network $[5,11,42,43]$ was reproduced. The basal ganglia network was also found to be anticorrelated with the Default Mode, and correlated, to a similar degree, with the lateralised attention networks. Although we and others have shown that RSNs possess similar frequency characteristics, it must be the case that they are at most weakly coupled, or they would not be separable in functional connectivity analyses [47]. Correlation values observed here between component time courses are correspondingly low - in the range of 0.19 to 0.27 in magnitude - consistent with those observed in other studies [12]. Despite the fact that they are weak, their consistency across subjects is such that these results are highly significant. There have been suggestions that the thalamus, with involvement of the basal ganglia, may be responsible for instigating the task-independent deactivation of the Default Mode observed when subjects are posed cognitively demanding tasks [48]. This would be consistent with findings by Uddin et al., which have shown that correlations between homologous RSN structures in the cerebral hemispheres are preserved in a patient with complete commissurotomy, indicating that functional connectivity can be mediated by subcortical structures [49]. The hypothesis that the basal ganglia RSN represents the controller of fluctuations in the Default mode is supported by the results of the functional network connectivity analysis. In 18 out the 20 randomly composed groups of 13 subjects, the time course of the basal ganglia independent component preceded that of the Default Mode. Although this demonstrates that the hemodynamic response in the basal ganglia RSN consistently precedes that in the Default Mode, latency differences in the hemodynamic response functions [50] in both networks would have to be analysed and corrected for [51] before concluding that activation in the basal gan- 
glia network precedes deactivation in the Default Mode. Possible differences between the precedence of neuronal activation and the measured MR response arising from hemodynamic shape and latency effects [52] preclude testing order hypotheses with this and other approaches such as Granger Causality Modeling.

Although RSNs show a maximum in frequency spectra in the range $0.01-0.04 \mathrm{~Hz}$, this does not accurately reflect the frequency distribution of underlying neuronal fluctuations. The intrinsic autocorrelation of BOLD fMRI data has 1 /f behaviour in the frequency domain [53], and low frequencies are cut off by sampling over a finite duration, leading to a low frequency peak. In fact, when the hemodynamic response function is deconvolved from RSN time courses prior to frequency analysis, the spectra of RSN components are essentially flat up to $0.1 \mathrm{~Hz}$ [27]. The analysis of RSN frequencies in this work serves two purposes, neither of which relate to the absolute frequencies observed in the spectra. The first is that differences between the compositions of RSN spectra can be observed. The frequency spectrum of the basal ganglia network is most similar in composition to that of the Default Mode, reinforcing the connection between the two networks. The second is that RSN spectra may be distinguished from components of physiological origin because their frequency distributions reflect the intrinsic convolution with the hemodynamic response function. This is the basis for the classifier used here, which can reliably distinguish RSNs from physiological components using frequency characteristics alone [54]. The basal ganglia RSN clusters clearly with other RSNs.

It is likely that the component which consists solely of the caudate (Figure 3 ) reflects activity relating to the inhibition of ocular saccades. As such, caudate activity is a taskspecific response rather than an RSN. The caudate component is apparent in the fixation study, in which there was a point fixation condition, while no corresponding activity is apparent in the eyes closed study. The role of the caudate in saccadic eye movements is well documented [5557]. Fluctuations in caudate activity in this capacity (which are a pre-requisite of their identification in an ICA) may arise due to phasic preparation of reflexive saccades and their voluntary inhibition. Ultimately controlled by the superior colliculus, saccades can be generated and inhibited by the input of the caudate nucleus, via efferent projections to the substantia nigra pars reticulata [57]. The oculomotor basal ganglia-thalamocortical circuit, includes, as origins of input to the caudate, frontal eye fields, dorsolateral prefrontal cortex and posterior parietal cortex (Brodmann's areas 8,9\&10 and 7, respectively) [36] all of which areas are apparent in this component. Frontal eye field neurons are known to fire during passive fixation [58] as was the condition in the fixation study. The oculomotor network yielded from the dorsal caudate seed in the Di Martino et al. analysis [31] is similar to the caudate component identified here in the fixation study. Our hypothesis that this is a task-related response to the suppression of ocular saccades is consistent with the use of a fixation condition in that work, and it not being observed in the many previous studies which have used the eyes closed condition [9,21-23,32].

Another area of application of this network is as a candidate marker for neuro- and psychopathologies involving the basal ganglia. In a parallel with attempts to use Default Mode activity as a diagnostic marker for Alzheimer's disease [8], deficits in the basal ganglia RSN may offer a marker for one or more of the diseases in which the basal ganglia are known to play a role. Parkinson's disease is a basal ganglia disorder characterized by the degeneration of dopaminergic neurons in the striatum. This has been shown to have opposing effects on the direct and indirect pathways in the striatum [59], with hyperkinesia as a result. Functional connectivity in the basal gangliacortical circuit has been demonstrated in this condition via synchronous oscillations between local field potentials in the basal ganglia and cortical EEG [60], the transmission to the basal ganglia of motor cortex electrostimulation in the monkey and rat [61] and oscillatory high-voltage spindles)[62]. Dopaminergic lesion in a rodent model for Parkinson's has been shown to lead to an increase in oscillatory synchronisation in the basal ganglia and increase in frequency and duration of high voltage spindle events)[62]. Similarly, excessive synchronisation of subthalamic nucleus neurons has been confirmed as a cause of movement slowing in Parkinsonism [63]. Dopamine-dependent changes in the functional connectivity between the basal ganglia and cortex have likewise been demonstrated [64]. The discovery of the fMRI manifestation of this functional connectivity will allow dysfunction in this system to be probed non-invasively, even if patients are not able to perform motor tasks appropriately.

\section{Conclusion}

We report the existence of a resting state in the thalamus and basal ganglia, showing greatest activity in the putamen, pallidum, substantia nigra and subthalamic nucleus, with projections to transverse temporal gyrus and the supplementary motor areas. The network is consistent with the cortico-subcortical motor control circuit of the basal ganglia and is robustly reproducible across subjects, scanning parameters and common behavioural conditions for resting state studies. The frequency spectrum of this component peaks at $0.025 \pm 0.007 \mathrm{~Hz}$ and is most similar in frequency composition to the Default Mode. Spectral features are similar to those of other resting state networks and distinct from physiological artefacts. Fluctu- 
ations in the basal ganglia network precede those in the Default Mode, with which it is anticorrelated. We posit that another independent component focussed in the caudate, which was observed in the fixation condition but not in the eyes closed condition, and which was recently reported as being associated with the resting state of the brain [31], is related to the suppression of ocular saccades.

The basal ganglia resting state network reported here offers the possibility to improve experiment design and analysis in fMRI studies of the striatum, and a possible window into disorders of the basal ganglia such as Parkinson's disease.

\section{Methods \\ MR data acquisition, fixation study (3 T)}

Twenty-six female right-handed subjects with no history of neurological or psychiatric disease and aged between 22 and 41 years (mean $26 \pm 5$ years) participated in the study, which was approved by the Ethics Committee of the Medical University of Vienna, with informed written consent. Magnetic resonance images were acquired with a 3 T Bruker Medspec S300 scanner (Bruker Biospin, Ettlingen, Germany) using a birdcage head coil. $\mathrm{T}_{1}$-weighted structural images were obtained with a 3D MPRAGE sequence with $\mathrm{TE}=8 \mathrm{~ms}$; flip angle $=15^{\circ} ; \mathrm{TA}=13 \mathrm{~min}$. For the resting state run, which was of 5 minutes duration, subjects were asked to fixate on a point projected onto a screen mounted in the scanner bore, which they viewed via a mirror attached to the head resonator. They were instructed not to engage in organised thought and not to sleep. Compliance was established verbally on completion of the experiment. Resting state EPI data was acquired with 18 oblique slices (parallel to a line defined by the anterior and posterior commissures (ACPC)) of $4 \mathrm{~mm}$ thickness with a $1 \mathrm{~mm}$ nominal gap. Other imaging parameters were as follows: TE/TR $=28 / 1000 \mathrm{~ms}$, a matrix size of $64 \times 64$ and a field of view of $21 \times 25 \mathrm{~cm}^{2}$ (left-right and anterior-posterior, respectively) yielding $3.3 \times 3.9 \times 4$ $\mathrm{mm}^{3}$ voxels, $\mathrm{NR}=300, \mathrm{TA}=5 \mathrm{~min}$. Imaging was prefaced by $10 \mathrm{~s}$ of dummy scans to ensure a steady state of longitudinal magnetisation.

\section{MR data acquisition, eyes closed study (4 T)}

A second group of subjects was studied in the eyes closed condition. Fifteen subjects ( 8 males) with no history of neurological or psychiatric disease and aged between 19 and 56 years, mean $36 \pm 12$ years, participated in the study, which was approved by the Ethics Committee of the University of Trento, with informed written consent. Magnetic resonance images were acquired with a $4 \mathrm{~T}$ Bruker Medspec scanner (Bruker Medical, Ettlingen, Germany) using a birdcage-transmit, eight-channel receive head coil (USA Instruments, Inc., Ohio, USA). $\mathrm{T}_{1^{-}}$ weighted structural images were obtained using a 3D
MPRAGE sequence with TE $=4 \mathrm{~ms}$; flip angle $=7^{\circ} ; \mathrm{iPAT}$ factor $2, \mathrm{TA}=5 \mathrm{~min}$ optimized for maximal contrast to noise ratio between grey and white matter at $4 \mathrm{~T}$ [65]. Subjects were asked to close their eyes during two resting state runs of 10 minutes duration each, and not engage in organised thought and not sleep (compliance established verbally as in the fixation study). EPI data were acquired along the ACPC line, with 37 oblique slices of $3 \mathrm{~mm}$ thickness with a $0.5 \mathrm{~mm}$ nominal gap, TE/TR $=33 / 2200$ ms, a matrix size of $64 \times 64$ and $192 \times 192 \mathrm{~mm}$, yielding $3 \times 3 \times 3 \mathrm{~mm}$ voxels. 273 volumes plus 2 preparation scans were acquired in a 10 min session. Resting state runs were prefaced by a point-spread function acquisition to allow the correction of geometric distortions [66], which has been demonstrated to be effective at high field [67].

\section{Data Analysis}

In the eyes closed study, distortion correction of EPI data was performed online using the point-spread function method as implemented in Siemens Distortion Correction WIP Version 2.5 [66]. Functional data acquired in both studies were preprocessed using SPM5 (motion correction, normalisation to the EPI template, no spatial or temporal smoothing). For both the fixation and control studies, Probabilistic Independent Component Analysis (PICA) was performed with MELODIC Version 3.05, part of FSL 4.0 [68]. The multi-session temporal concatenation group ICA approach was employed in order to find common spatial patterns amongst subjects without assuming similarity in temporal responses. Whilst the same approach is employed in the Group ICA of FMRI Toolbox (GIFT) [20], we used MELODIC to estimate component spatial maps in order both to facilitate comparison with the majority of studies which have looked for RSNs common to healthy subjects $[21-23,32]$ and because probabilistic approach allows alternative hypothesis testing of the significance of activated voxels [18]. Downsampling of data is common in group ICA to reduce total data volume and to increase SNR. Here we analysed data at two resolutions - downsampled to $4 \mathrm{~mm}$ and also with no downsampling, the latter to try to resolve activation in small structures. In the PICA analysis masking was applied to exclude non-brain voxels. The data were de-meaned on a voxel-by-voxel basis, and the voxel-wise variance normalised. The number of components into which the data were decomposed was determined using the Laplace approximation to the Bayesian evidence for a probabilistic principal component analysis model implemented in MELODIC [18]. Estimated component maps were divided by the standard deviation of the residual noise. Statistical significance was attributed by fitting a mixture model to the histogram of intensity values [18]. To establish the reproducibility of responses the same analysis was applied to two equally sized, randomly selected, non-overlapping subgroups of 13 subjects in the fixation study; Subgroup 
1 , (mean age $25 \pm 3$ years) and Subgroup 2 (mean age 27 \pm 6 years) (age difference not significant in student's t-test; $\mathrm{P}=0.37)$.

Anatomical connectivity determines function [69]. The likelihood of brain regions being connected may be determined from diffusion weighted imaging data in an approach known as probabilistic diffusion tractography [41], which can provide useful insight into the roles of RSNs [21]. The probabilities of activation foci in the thalamus connecting to particular cortical regions were assessed using an open-access probabilistic diffusion tractography atlas http://www.fmrib.ox.ac.uk/connect/ based on the results of a study by Behrens et al. [41].

Data in the fixation study were additionally analysed with GIFT [20]. GIFT employs a temporal concatenation approach like that used in this MELODIC analysis, but also back-reconstructs single-subject spatial maps in addition to time courses. In our experience, the temporal responses of single subjects are better separated by GIFT, and we used these as the basis for analysis of temporal features, including characterisation of the frequency distributions of RSNs, the temporal relationships between RSNs (correlations between component time courses) and the attempt to distinguish RSNs and physiological components using temporal features. These further analyses were performed using MATLAB (Mathworks Inc, Natick, MA) routines developed in-house.

Frequency spectra were calculated from component time courses using Welch's averaged, modified periodogram spectral estimation method, using a Hamming window over periods of $64 \mathrm{~s}$, with $50 \%$ overlap between segments. The peak frequency was calculated as the mean frequency over subjects at which the spectral power was a maximum.

To investigate correlations between component time courses (which may indicate functional relationships between networks $[11,42])$ the correlation coefficients between all possible pairs of component time courses were calculated. To avoid the possibility of introducing anticorrelations between networks $[25,27,28]$, no global signal subtraction was performed. Fischer transformation was applied to single-subject correlation values (to normalise their distribution), and the null hypothesis of no correlation between the time courses tested with one-sample t tests at the $\mathrm{P}<0.05$ level.

An extension of the approach of correlating RSN time courses, termed Functional Network Connectivity [12], has been developed to allow the study of not only the correlation between networks, but also which leads and which lags in response. The functional network connectivity toolbox http://mialab.mrn.org/ was applied to GIFT results. In keeping with the identification of the peak in spectral power at $0.025 \pm 0.007 \mathrm{~Hz}$, correlations were assessed in the frequency range 0.01 to $0.4 \mathrm{~Hz}$. Only lags between components reflecting the Default Mode and the basal ganglia resting state network were assessed, in view of the hypothesised connection between the two networks. A significance threshold of $\mathrm{P}<0.05$ was set for correlations. As an additional means to assess the reliability of calculated correlations and lags, the analysis was performed for each of 20 randomly-composed groups of 13 subjects from the population of 26 subjects.

Given differences in the frequency composition of RSN and physiological components (see [29] and Results section here), two features suggested themselves as the basis for a classifier. These have been labelled "Dynamic Range" - the difference in power between the maximum and the minimum of the distribution, and "Low to High Power Ratio" - the ratio of the integral of power in the region of the spectrum below $0.02 \mathrm{~Hz}$ to the total. Spectra were smoothed with MATLAB's digital filter function ([70] pp311-2), with the filter described by numerator coeffi-

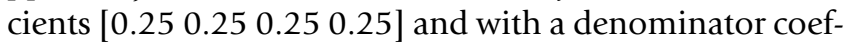
ficient of 1 , prior to the calculation of features, which were evaluated for each subject and component and plotted in the feature space. Discrimination thresholds were established using the iterative threshold selection algorithm described in Ridler and Calvard [71] in which the threshold starting point $\theta$ is the mean value of the entire bimodal distribution and the value of the threshold for the $\mathrm{k}^{\text {th }}$ iteration is

$$
\theta_{k}=\frac{\left(m_{s, k-1}+m_{i, k-1}\right)}{2}
$$

where $\mathrm{m}_{\mathrm{s}, \mathrm{k}-1}, \mathrm{~m}_{\mathrm{i}, \mathrm{k}-1}$ are the means of the distributions inferior and superior to the threshold for the previous iteration. This was performed until $\theta_{\mathrm{k}}=\theta_{\mathrm{k}-1}$. To avoid introducing bias, the basal ganglia component was not included in the development of the classifier or the establishment of discrimination thresholds.

The most salient details of the two study groups and the analysis methods applied to each are shown in a data analysis flow chart, Additional File 4.

\section{Authors' contributions}

SR conceived of the study, acquired the fixation $\mathrm{AMRI}$ data, performed the MELODIC ICA and FNC analyses, developed the interpretation and wrote the manuscript. US recruited and tested subjects and reviewed possible alternative interpretations of the network's function. GB acquired the eyes closed data, performed neuroanatomical labelling and assisted with interpretation. NS performed the GIFT analysis, frequency and correlation 
analyses and developed the classifier. JJ and LB guided the analysis of temporal and frequency features and the classifier and IKE, HB and EM contributed to interpretation of the network's function. All authors read and approved the final manuscript.

\section{Additional material}

\section{Additional file 1}

Independent component for the basal ganglia RSN (MELODIC). This image is of the independent component for the basal ganglia identified in the MELODIC analysis, thresholded at $P>0.5$ (downsampled data). Click here for file

[http://www.biomedcentral.com/content/supplementary/14712202-10-137-S1.PNG]

\section{Additional file 2}

Independent component for the basal ganglia RSN (GIFT). This image is of the independent component for the basal ganglia identified in the GIFT analysis (downsampled data).

Click here for file

[http://www.biomedcentral.com/content/supplementary/14712202-10-137-S2.PNG]

\section{Additional file 3}

Hybrid simulations, ICA separation of spatially overlapping components. This document contains text and images describing hybrid simulation showing that ICA is capable of separating circuits which overlap to some extent, but which also have non-overlapping elements and different temporal behaviour.

Click here for file

[http://www.biomedcentral.com/content/supplementary/14712202-10-137-S3.PDF]

\section{Additional file 4}

Analysis flow chart. This image shows the most important details relating to the two study groups and the analysis methods applied to each, presented as a flowchart.

Click here for file

[http://www.biomedcentral.com/content/supplementary/14712202-10-137-S4.PNG]

\section{Acknowledgements}

This study was supported by the government of the Provincia Autonoma di Trento, Italy, the private foundation Fondazione Cassa di Risparmio di Trento e Rovereto, the University of Trento, Italy, grant I I 437 from the Austrian National Bank and by a Siemens Medical Solutions research grant to EM.

\section{References}

I. Biswal B, Yetkin FZ, Haughton VM, Hyde JS: Functional connectivity in the motor cortex of resting human brain using echoplanar MRI. Magn Reson Med 1995, 34(4):537-54I.

2. Biswal BB, Van Kylen J, Hyde JS: Simultaneous assessment of flow and BOLD signals in resting-state functional connectivity maps. NMR Biomed 1997, 10(4-5): 165-170.

3. Cordes D, Haughton VM, Arfanakis K, Carew JD, Turski PA, Moritz $\mathrm{CH}$, Quigley MA, Meyerand ME: Frequencies contributing to functional connectivity in the cerebral cortex in "restingstate" data. AJNR Am J Neuroradiol 200I, 22(7): I326-1333.
4. Lowe MJ, Mock BJ, Sorenson JA: Functional connectivity in single and multislice echoplanar imaging using resting-state fluctuations. Neurolmage 1998, 7(2): I 19-132.

5. Greicius M, Krasnow B, Reiss A, Menon V: Functional connectivity in the resting brain: a network analysis of the default mode hypothesis. Proc Natl Acad Sci USA 2003, I 00( I):253-258.

6. Fox MD, Snyder AZ, Zacks JM, Raichle ME: Coherent spontaneous activity accounts for trial-to-trial variability in human evoked brain responses. Nat Neurosci 2006, 9(I):23-25.

7. Fox MD, Snyder AZ, Vincent JL, Raichle ME: Intrinsic fluctuations within cortical systems account for intertrial variability in human behavior. Neuron 2007, 56(I): I7I-I84.

8. Greicius MD, Srivastava G, Reiss AL, Menon V: Default-mode network activity distinguishes Alzheimer's disease from healthy aging: evidence from functional MRI. Proc Natl Acad Sci USA 2004, I 0 I(13):4637-4642.

9. Calhoun VD, Kiehl KA, Pearlson GD: Modulation of temporally coherent brain networks estimated using ICA at rest and during cognitive tasks. Hum Brain Mapp 2008, 29(7):828-838.

10. Morcom AM, Fletcher PC: Does the brain have a baseline? Why we should be resisting a rest. Neuroimage 2007, 37(4): 1073-1082.

II. Fox MD, Snyder AZ, Vincent JL, Corbetta M, Van Essen DC, Raichle ME: The human brain is intrinsically organized into dynamic, anticorrelated functional networks. Proc Natl Acad Sci USA 2005, I 02(27):9673-9678.

12. Jafri MJ, Pearlson GD, Stevens M, Calhoun VD: A method for functional network connectivity among spatially independent resting-state components in schizophrenia. Neurolmage 2008, 39(4): 1666-168I.

13. Biswal BB, Yetkin FZ, Haughton VM, Hyde JS: Functional connectivity in the auditory cortex studied with FMRI. Neurolmage 1996, 3:S305.

14. Stein T, Moritz C, Quigley M, Cordes D, Haughton V, Meyerand E: Functional connectivity in the thalamus and hippocampus studied with functional MR imaging. AJNR Am J Neuroradiol 2000, 2I (8): |397-| |40|.

15. Shulman G, Fiez J, Corbetta M, Buckner R, Miezin F, Raichle M, Petersen S: Common blood flow changes across visual tasks: II. Decreases in cerebral cortex. Journal of Cognitive Neuroscience 1997, 9(3):648-663.

16. Raichle M, MacLeod A, Snyder A, Powers W, Gusnard D, Shulman G: A default mode of brain function. Proc Natl Acad Sci USA 200I, 98(2):676-682.

17. Mazoyer B, Zago L, Mellet E, Bricogne S, Etard O, Houde O, Crivello F, Joliot M, Petit L, Tzourio-Mazoyer N: Cortical networks for working memory and executive functions sustain the conscious resting state in man. Brain Res Bull 200I, 54(3):287-298.

18. Beckmann CF, Smith SM: Probabilistic independent component analysis for functional magnetic resonance imaging. IEEE Trans Med Imaging 2004, 23(2): I37-I52.

19. Beckmann CF, Smith SM: Tensorial extensions of independent component analysis for multisubject FMRI analysis. Neurolmage 2005, 25(I):294-3II.

20. Calhoun VD, Adali T, Pearlson GD, Pekar J]: A method for making group inferences from functional MRI data using independent component analysis. Hum Brain Mapp 200I, I4(3):I40-I5I.

21. Beckmann CF, DeLuca M, Devlin JT, Smith SM: Investigations into resting-state connectivity using independent component analysis. Philos Trans $R$ Soc Lond B Biol Sci 2005, 360(1457): $1001-1013$.

22. De Luca M, Beckmann CF, De Stefano N, Matthews PM, Smith SM: fMRI resting state networks define distinct modes of longdistance interactions in the human brain. Neurolmage 2006, 29(4): 1359-1367.

23. Damoiseaux JS, Rombouts SA, Barkhof F, Scheltens P, Stam CJ, Smith SM, Beckmann CF: Consistent resting-state networks across healthy subjects. Proc Natl Acad Sci USA 2006, 103(37): | 3848-| 3853.

24. Achard S, Salvador R, Whitcher B, Suckling J, Bullmore E: A resilient, low-frequency, small-world human brain functional network with highly connected association cortical hubs. I Neurosci 2006, 26(I):63-72.

25. Weissenbacher A, Kasess C, Gerstl F, Lanzenberger R, Moser E, Windischberger $C$ : Correlations and anticorrelations in resting-state functional connectivity MRI: a quantitative com- 
parison of preprocessing strategies. Neurolmage 2009 , 47(4): $1408-14 \mid 6$

26. Birn RM, Diamond JB, Smith MA, Bandettini PA: Separating respiratory-variation-related fluctuations from neuronal-activityrelated fluctuations in fMRI. Neurolmage 2006, 3 I(4): I536- I 548.

27. Smith S, Niazy R, Beckmann C, Miller K: Resting State Networks - Neither Low Frequency Nor Anticorrelated? Neurolmage 2008, 4 I (Supplement I):SI8I-227.

28. Murphy K, Birn RM, Handwerker DA, Jones TB, Bandettini PA: The impact of global signal regression on resting state correlations: are anti-correlated networks introduced? Neurolmage 2009, 44(3):893-905.

29. Birn RM, Murphy K, Bandettini PA: The effect of respiration variations on independent component analysis results of resting state functional connectivity. Hum Brain Mapp 2008, 29(7):740-750.

30. Robinson S, Soldati N, Basso G, Sailer U, Jovicich J, Bruzzone LI, Kryspin-Exner I, Bauer H, Moser E: A Resting State Network in the Basal Ganglia. Proceedings of the Sixteenth Annual Meeting of the ISMRM, Toronto 2008.

31. Di Martino A, Scheres A, Margulies DS, Kelly AM, Uddin LQ, Shehzad Z, Biswal B, Walters JR, Castellanos FX, Milham MP: Functional connectivity of human striatum: a resting state FMRI study. Cereb Cortex 2008, 18( ( 2):2735-2747.

32. Damoiseaux JS, Beckmann CF, Arigita EJ, Barkhof F, Scheltens P, Stam C], Smith SM, Rombouts SA: Reduced resting-state brain activity in the "default network" in normal aging. Cereb Cortex 2008, | 8(8): | 1856-| 864 .

33. Logothetis NK, Murayama $Y$, Augath M, Steffen T, Werner J, Oeltermann A: How not to study spontaneous activity. Neurolmage 2009, 45(4): 1080-1089.

34. Kandel ER, Schwartz JH, Jessell TM: Principles of Neural Science 4th edition. New York: McGraw-Hill; 2000.

35. Kunzle H: An autoradiographic analysis of the efferent connections from premotor and adjacent prefrontal regions (areas 6 and 9) in macaca fascicularis. Brain, behavior and evolution 1978, I5(3): 185-234.

36. Alexander GE, DeLong MR, Strick PL: Parallel organization of functionally segregated circuits linking basal ganglia and cortex. Annu Rev Neurosci 1986, 9:357-38I.

37. Yelnik J: Functional anatomy of the basal ganglia. Mov Disord 2002, 17(Suppl 3):SI 5-2I.

38. Robinson S, Moser E, Peper M: fMRI of Emotion. In fMRI Techniques and Protocols Volume 4I. Edited by: Filippi M. New Jersey: Humana Press; 2009.

39. Robinson S, Pripfl J, Bauer H, Moser E: The impact of EPI voxel size on SNR and BOLD sensitivity in the anterior mediotemporal lobe: a comparative group study of deactivation of the Default Mode. MAGMA 2008, 2 I (4):279-290.

40. Rombouts SA, Damoiseaux JS, Goekoop R, Barkhof F, Scheltens P, Smith SM, Beckmann CF: Model-free group analysis shows altered BOLD FMRI networks in dementia. Hum Brain Mapp 2009, 30(I):256-266.

4I. Behrens TE, Johansen-Berg H, Woolrich MW, Smith SM, WheelerKingshott CA, Boulby PA, Barker G], Sillery EL, Sheehan K, Ciccarelli $O$, et al.: Non-invasive mapping of connections between human thalamus and cortex using diffusion imaging. Nat $\mathrm{Neu}$ rosci 2003, 6(7):750-757.

42. Fransson P: Spontaneous low-frequency BOLD signal fluctuations: an fMRI investigation of the resting-state default mode of brain function hypothesis. Hum Brain Mapp 2005, 26(I): I5-29.

43. Hampson M, Peterson BS, Skudlarski P, Gatenby JC, Gore JC: Detection of functional connectivity using temporal correlations in MR images. Hum Brain Mapp 2002, I 5(4):247-262.

44. Slavin KV, Thulborn KR, Wess C, Nersesyan H: Direct visualization of the human subthalamic nucleus with $3 T$ MR imaging. AJNR Am / Neuroradiol 2006, 27(I):80-84.

45. Zhang D, Snyder AZ, Fox MD, Sansbury MW, Shimony JS, Raichle ME: Intrinsic functional relations between human cerebral cortex and thalamus. I Neurophysiol 2008, I 00(4): 1740-1748.

46. Gelman N, Gorell JM, Barker PB, Savage RM, Spickler EM, Windham JP, Knight RA: MR imaging of human brain at 3.0 T: preliminary report on transverse relaxation rates and relation to estimated iron content. Radiology 1999, 2 10(3):759-767.

47. Calhoun VD, Adali T, Pekar J], Pearlson GD: Latency (in)sensitive ICA. Group independent component analysis of fMRI data in the temporal frequency domain. Neurolmage 2003, 20(3): $1661-1669$

48. Gusnard DA, Raichle ME, Raichle ME: Searching for a baseline: functional imaging and the resting human brain. Nat Rev Neurosci $200 I, 2(10): 685-694$.

49. Uddin LQ, Mooshagian E, Zaidel E, Scheres A, Margulies DS, Kelly AM, Shehzad Z, Adelstein JS, Castellanos FX, Biswal BB, et al.: Residual functional connectivity in the split-brain revealed with resting-state functional MRI. Neuroreport 2008, 19(7):703-709.

50. Handwerker DA, Ollinger JM, D'Esposito M: Variation of BOLD hemodynamic responses across subjects and brain regions and their effects on statistical analyses. Neurolmage 2004, 2I(4): |639-|65|

51. Chang C, Thomason ME, Glover GH: Mapping and correction of vascular hemodynamic latency in the BOLD signal. Neurolmage 2008, 43(I):90-102.

52. Friston K: Causal modelling and brain connectivity in functional magnetic resonance imaging. PLoS biology 2009, 7(2):e33.

53. Zarahn E, Aguirre GK, D'Esposito M: Empirical analyses of BOLD fMRI statistics. I. Spatially unsmoothed data collected under null-hypothesis conditions. Neurolmage 1997, 5(3): 179-197.

54. Soldati N, Robinson S, Persello C, Jovicich J, Bruzzone L: Automatic classification of brain resting states using fMRI temporal signals. Electronics Letters 2009, 45(I): 19-2I

55. Scholz VH, Flaherty AW, Kraft E, Keltner JR, Kwong KK, Chen YI, Rosen BR, Jenkins BG: Laterality, somatotopy and reproducibility of the basal ganglia and motor cortex during motor tasks. Brain Res 2000, 879(I-2):204-2I5.

56. Hikosaka O, Sakamoto M, Usui S: Functional properties of monkey caudate neurons. I. Activities related to saccadic eye movements. J Neurophysiol 1989, 6 I (4):780-798.

57. Hikosaka O, Wurtz RH: The basal ganglia. Reviews of oculomotor research 1989, 3:257-281.

58. Mohler CW, Goldberg ME, Wurtz RH: Visual receptive fields of frontal eye field neurons. Brain Res 1973, 61:385-389.

59. Mallet N, Ballion B, Le Moine C, Gonon F: Cortical inputs and GABA interneurons imbalance projection neurons in the striatum of parkinsonian rats. J Neurosci 2006, 26(14):3875-3884.

60. Lalo E, Thobois S, Sharott A, Polo G, Mertens P, Pogosyan A, Brown $P$ : Patterns of bidirectional communication between cortex and basal ganglia during movement in patients with Parkinson disease. J Neurosci 2008, 28( I 2):3008-30I6.

61. Kolomiets BP, Deniau JM, Glowinski J, Thierry AM: Basal ganglia and processing of cortical information: functional interactions between trans-striatal and trans-subthalamic circuits in the substantia nigra pars reticulata. Neuroscience 2003, I I 7(4):93I-938.

62. Dejean C, Gross CE, Bioulac B, Boraud T: Dynamic changes in the cortex-Basal Ganglia network after dopamine depletion in the rat. I Neurophysiol 2008, I00(I):385-396.

63. Chen CC, Litvak V, Gilbertson T, Kuhn A, Lu CS, Lee ST, Tsai CH, Tisch S, Limousin P, Hariz M, et al.: Excessive synchronization of basal ganglia neurons at $20 \mathrm{~Hz}$ slows movement in Parkinson's disease. Experimental neurology 2007, 205(I):2I4-22I.

64. Williams D, Tijssen M, Van Bruggen G, Bosch A, Insola A, Di Lazzaro V, Mazzone P, Oliviero A, Quartarone A, Speelman H, et al: Dopamine-dependent changes in the functional connectivity between basal ganglia and cerebral cortex in humans. Brain 2002, 125(Pt 7): 1558-1569.

65. Papinutto $\mathrm{N}$, Jovicich J: Optimization of brain tissue contrast in structural images at 4T: a computer simulation and validation study. 25th Congress of the European Society for Magnetic Resonance in Medicine and Biology. Valencia, Spain 2008.

66. Zaitsev M, Hennig J, Speck O: Point spread function mapping with parallel imaging techniques and high acceleration factors: fast, robust, and flexible method for echo-planar imaging distortion correction. Magn Reson Med 2004, 52(5): I1 $156-1166$

67. Robinson S, Jovicich J: EPI distortion corrections at 4T: multichannel field mapping and a comparison with the pointspread function method. Proceedings of the Sixteenth Annual Meeting of the ISMRM, Toronto 2008.

68. Smith SM, Jenkinson M, Woolrich MW, Beckmann CF, Behrens TE, Johansen-Berg H, Bannister PR, De Luca M, Drobnjak I, Flitney DE, et al: Advances in functional and structural MR image analysis 
and implementation as FSL. Neurolmage 2004, 23(Supplement I):S208-219.

69. Passingham RE, Stephan KE, Kotter R: The anatomical basis of functional localization in the cortex. Nat Rev Neurosci 2002, 3(8):606-616.

70. Oppenheim AVaS RW: Discrete-Time Signal Processing Englewood Cliffs, NJ: Prentice-Hall; 1989.

71. Ridler TW, Calvard S: Picture thresholding using an iterative selection method. IEEE Transactions on Systems, Man and Cybernetics 1978, 8:630-632.

Publish with Bio Med Central and every scientist can read your work free of charge

"BioMed Central will be the most significant development for disseminating the results of biomedical research in our lifetime." Sir Paul Nurse, Cancer Research UK

Your research papers will be:

- available free of charge to the entire biomedical community

- peer reviewed and published immediately upon acceptance

- cited in PubMed and archived on PubMed Central

- yours - you keep the copyright
BioMedcentral 